\section{HIV suppression by interleukin-16}

SIR - In addition to their function as cytotoxic T cells, lymphocytes carrying the CD8 receptor $\left(\mathrm{CD}^{+}\right)$secrete one or more soluble factors which inhibit replication of HIV and SIV (human and simian immunodeficiency viruses, respectively) in primary $\mathrm{CD}^{+}{ }^{+}$cells ${ }^{1,2}$. This antiviral activity is positively correlated with the health of HIVinfected patients, and is high during the asymptomatic stages of infection. Old World monkeys such as the African green monkey Cercopithecus aethiops, which are naturally infected with SIV in the wild, possess high immunodeficiency virus-suppressing lymphokine (ISL) activity ${ }^{2}$, a low viral load 3 , and never develop simian AIDS $^{4}$.

Here we show that the previously identified lymphocyte chemoattractant, interleukin-16 (IL-16), which is secreted from activated $\mathrm{CD} 8^{+}$cells and binds to T cells through the CD4 receptor ${ }^{5,6}$, suppresses the replication of HIV and SIV. The IL-16 from African green monkeys is highly homologous to its human counterpart. It may therefore contribute to the low viral load in healthy HIV-infected patients and naturally infected African green monkeys.

The IL-16 molecules isolated from African green monkeys and humans (Fig. 1) are proteins of 130 amino acids and relative molecular mass around 13,500. The genes encoding human (HU) and African green monkey (AGM) IL-16 differ in 16 of their 390 coding nucleotides, resulting in 7 non-clustered amino-acid changes.

On analysis by gel filtration, recombinant soluble IL-16 $6_{\mathrm{HU}}$ and IL-16 $6_{\mathrm{AGM}}$ elute predominantly as homodimers (apparent $M_{\mathrm{r}} 28,000$ ), with minor peaks corresponding to monomeric and tetrameric forms. The lymphocyte chemoattractant activity of IL-16 was previously linked to its tetramer formation ${ }^{5}$, so it is possible that a

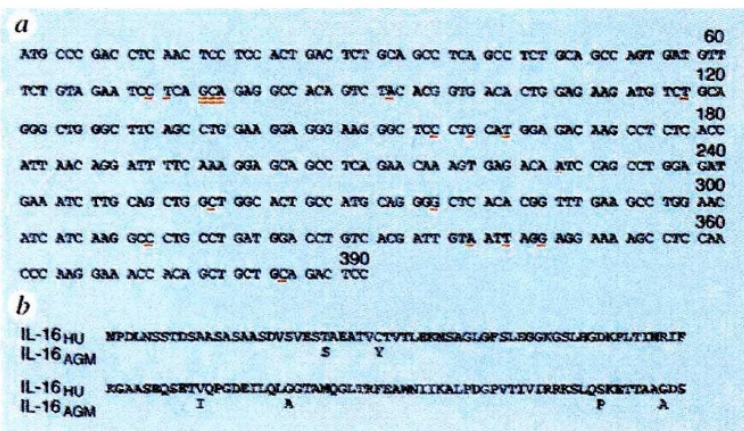

FIG. 1 a, Nucleotide sequence of IL-16 ${ }_{\text {AGM. }}$. Differences from the human IL-16 sequence are underlined. Codon 26 , which is deleted in some of the human and African green monkey IL-16 alleles, is marked by a double line. $b$, Alignment of the deduced aminoacid sequences of IL-16 $6_{\mathrm{H}}$ and IL-16 clear cells (PBMCs; $5 \times 10^{6}$ ) were cultivated with $10 \mu \mathrm{g} \mathrm{ml}^{-1}$ concanavalin A and IL-2 for $48 \mathrm{~h}$. Total RNA was prepared and a cDNA synthesis was performed in a standard polymerase chain reaction. Purified products were cloned for nucleotide sequence confirmation and protein expression into the vector $\mathrm{pET} 15 \mathrm{~b}$ (Novagen). Expressed IL-16 was obtained by $\mathrm{Ni}^{2+}-\mathrm{NTA}$ agarose (Qiagen) chromatography to $>95 \%$ purity. by the long terminal repeats of HIV without affecting initial binding by the virus . $^{8}$ Further investigation is needed to determine what fraction of the ISL activity is due to IL-16, but it has not escaped our attention that IL-16 may possess an antiviral therapeutic effect.

Michael Baier, Albrecht Werner
Norbert Bannert, Karin Metzner

Reinhard Kurth

Paul-Ehrlich-Institut,

Paul-Ehrlich-Strasse 51-59,

D-63225 Langen, Germany

1. Levy, J. A. AIDS 7, 1401-1410 (1993).

2. Ennen, J. et at. Proc. natn. Acad. Sci. U.S.A. 91 7207-7211 (1994)

3. Hartung, S. et al. J. Virol. 66, 2143-2149 (1992).

4. Norley, S. G. et al. Proc. natn. Acad. Sci. U.S.A. 87, 9067-9071 (1990).

5. Cruikshank, W. W. et al. Proc. natn. Acad. Sci. U.S.A 91, 5109-5113 (1994)

6. Laberge, S. et al. J. Immun. 155, 2902-2910 (1995).

7. Cruikshank, W. W. et al. J. Immun. 138, 3817-3823 (1987).

8. Corbeau, P. et at. J. Immun. 150, 290-301 (1993).

M.B. and A.W. contributed equally to this study.

\section{Tetrodotoxin as a pheromone}

SIR - Tetrodotoxin (TTX) is one of the most potent marine toxins ${ }^{1}$, and so one possible physiological role is as a defence agent $t^{2,3}$. Here I report another function of TTX, as a male-attracting pheromone at the time of spawning.

Measurement of attracting activity of the puffer fish Fugu niphobles against TTX was conducted by using a Y-maze

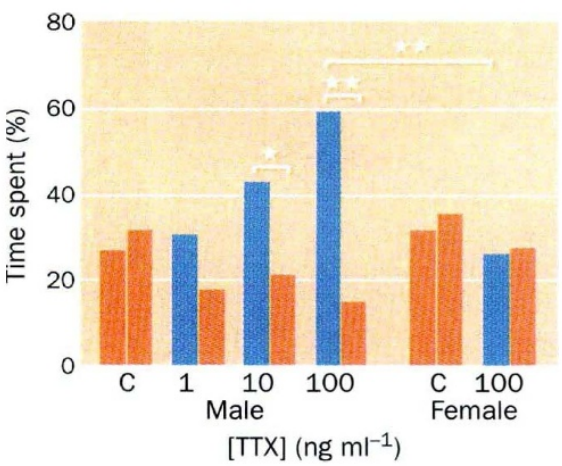

FIG. 1 Preference activities of $F$. niphobles, against $\Pi \mathrm{X}$. Results represent medians of eight males and females in each concentration. $* P<0.05 ; * * P<0.01$ when compared with control reactions. The preference reactions of spermating males and sexually mature females were tested by the Y-maze flow-through system ${ }^{4}$ operated after a fish was placed in the starting point at the centre of $Y$ maze. Artificial sea water was introduced into both arm inlets of the maze (dimensions $45 \times 8 \times 8 \mathrm{~cm}$ ) with a flow of 2,000 $\mathrm{ml} \mathrm{min}{ }^{-1}$. The $\Pi \mathrm{TX}$ solution and control distilled water were introduced into the arm channel in each inlet with a constant flow of $1.0 \mathrm{ml} \mathrm{min}^{-1}$. The percentage of the spent period $(5 \mathrm{~min}$ ) for the test fish in each channel was analysed statistically with Friedman's two-way analysis of variance, followed by Wilcoxon's signed-rank test or the Mann-Whitney U-test. Blue columns, TTX; pink columns, distilled water. 